\title{
Potential for Lung Recruitment and Ventilation-Perfusion Mismatch in Patients With the Acute Respiratory Distress Syndrome From Coronavirus Disease 2019*
}

\author{
Tommaso Mauri, $\mathrm{MD}^{1,2}$; Elena Spinelli, $\mathrm{MD}^{1}$; Eleonora Scotti, $\mathrm{MD}^{1}$; Giulia Colussi, $\mathrm{MD}^{1}$; \\ Maria Cristina Basile, $\mathrm{MD}^{1}$; Stefania Crotti, $\mathrm{MD}^{1}$; Daniela Tubiolo, $\mathrm{MD}^{1}$; Paola Tagliabue, $\mathrm{MD}^{1}$; \\ Alberto Zanella, $\mathrm{MD}^{1,2}$; Giacomo Grasselli, $\mathrm{MD}^{1,2}$; Antonio Pesenti, MD ${ }^{1,2}$
}

\begin{abstract}
Objectives: Severe cases of coronavirus disease 2019 develop the acute respiratory distress syndrome, requiring admission to the ICU. This study aimed to describe specific pathophysiological characteristics of acute respiratory distress syndrome from coronavirus disease 2019.
\end{abstract}

Design: Prospective crossover physiologic study.

Setting: ICU of a university-affiliated hospital from northern Italy dedicated to care of patients with confirmed diagnosis of coronavirus disease 2019.

Patients: Ten intubated patients with acute respiratory distress syndrome and confirmed diagnosis of coronavirus disease 2019. Interventions: We performed a two-step positive end-expiratory pressure trial with change of $10 \mathrm{~cm} \mathrm{H}_{2} \mathrm{O}$ in random order.

Measurements and Main Results: At each positive end-expiratory pressure level, we assessed arterial blood gases, respiratory mechanics, ventilation inhomogeneity, and potential for lung recruitment by electrical impedance tomography. Potential for lung recruitment was assessed by the recently described recruitment to inflation ratio. In a subgroup of seven paralyzed patients, we also measured ventilation-perfusion mismatch at lower positive end-expiratory pressure by electrical impedance tomography. At higher positive end-expiratory pressure, respiratory mechanics did not change significantly: compliance remained relatively high with low driving pressure. Oxygenation and ventilation inhomogeneity improved but arterial $\mathrm{CO}_{2}$ increased despite unchanged respiratory rate and tidal volume. The recruitment to inflation ratio presented median value higher than previously reported in acute respiratory distress syndrome patients but

\footnotetext{
*See also p. 1239.

'Department of Anesthesia, Critical Care and Emergency, Fondazione IRCCS Ca' Granda Ospedale Maggiore Policlinico, Milan, Italy.

${ }^{2}$ Department of Pathophysiology and Transplantation, University of Milan, Milan, Italy.

Copyright (C) 2020 by the Society of Critical Care Medicine and Wolters Kluwer Health, Inc. All Rights Reserved.
}

DOI: 10.1097/CCM.0000000000004386 with large variability (median, 0.79 [0.53-1.08]; range, 0.16-1.40). The $\mathrm{FIO}_{2}$ needed to obtain viable oxygenation at lower positive endexpiratory pressure was significantly correlated with the recruitment to inflation ratio $(r=0.603 ; p=0.05)$. The ventilation-perfusion mismatch was elevated (median, 34\% [32-45\%] of lung units) and, in six out of seven patients, ventilated nonperfused units represented a much larger proportion than perfused nonventilated ones.

Conclusions: In patients with acute respiratory distress syndrome from coronavirus disease 2019, potential for lung recruitment presents large variability, while elevated dead space fraction may be a specific pathophysiological trait. These findings may guide selection of personalized mechanical ventilation settings. (Crit Care Med 2020; 48:1129-1134)

Key Words: acute respiratory distress syndrome; coronavirus disease 2019; electrical impedance tomography; mechanical ventilation; positive end-expiratory pressure; pulmonary perfusion

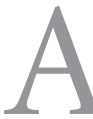
ccording to reports from China, about $20 \%$ of hospitalized patients $(1,2)$ and up to $70 \%$ of critically ill patients (2) with the novel coronavirus disease 2019 (COVID19) develop the acute respiratory distress syndrome (ARDS), which markedly increases the risk of death (3). The majority of patients with ARDS due to COVID-19 require intubation and invasive mechanical ventilation (1-3). Even though the overflow of patients due to the outbreak and the need for patients isolation limit the application of refined diagnostic procedures (4), bedside characterization of ARDS pathophysiology is essential to inform the management of these critically ill patients (5). Available data seem to indicate that ARDS due to COVID19 might present heterogeneous characteristics. The lung morphology is characterized by coexisting signs of alveolar damage and interstitial injury: ground-glass opacity with or without consolidation and septal thickening are common findings on CT images (6). Furthermore, progression of pulmonary injury 
is characterized by specific alterations of the pulmonary vasculature tree, with dynamic increase in the size of vessels $(7,8)$.

In this study, we assessed the respiratory mechanics, gas exchange, ventilation inhomogeneity, potential for lung recruitment, and ventilation/perfusion mismatch by electrical impedance tomography (EIT) in a cohort of intubated patients with ARDS from COVID-19. The study hypothesis was that heterogeneous findings could indicate the need for personalized treatment and that physiologic measures at low positive endexpiratory pressure (PEEP) might already yield useful guidance.

\section{MATERIALS AND METHODS}

\section{Study Population}

We conducted a prospective physiologic study on 10 intubated patients admitted to the general ICU of Maggiore Policlinico Hospital, Milan, Italy. Inclusion criteria were: intubated patients with confirmed infection by novel coronavirus 2019 (severe acute respiratory syndrome coronavirus 2) and diagnosis of ARDS on the day of the study, scheduled to undergo a PEEP trial for clinical reasons. Exclusion criteria were: age less than 18 years, hemodynamic instability, history of severe chronic obstructive pulmonary disease, pregnancy, contraindications to the use of EIT (e.g., presence of pacemaker or chest surgical wounds dressing). The ethical committee of the hospital approved the study (reference 239_2020) and informed consent was obtained according to local regulations.

\section{Data Collection}

After enrollment, we collected sex, age, body mass index, comorbidities, days of intubation, Sequential Organ Failure Assessment score, clinical PEEP level, and the severity of ARDS.

\section{EIT Monitoring}

We placed an EIT dedicated belt containing 16 electrodes around the patient's chest at the fifth or sixth intercostal space and connected it to an EIT monitor (PulmoVista 500; Dräger Medical GmbH, Lübeck, Germany). EIT data were generated by application of small alternate electrical currents rotating around patient's thorax, continuously registered at $50 \mathrm{~Hz}$ during all study phases and stored for offline analysis.

\section{Study Protocol}

Patients were connected to a ventilator (Servo I; Getinge AB, Göteborg, Sweden) and ventilation mode (seven on volumecontrolled ventilation, three on pressure support ventilation), tidal volume $\left(V_{T}\right)$, support, and respiratory rate (RR) were left as selected by the attending physician throughout the whole study. All patients were deeply sedated and kept in the semirecumbent position, seven patients on controlled ventilation were also paralyzed.

The study consisted of two crossover steps (i.e., all patients undergoing both phases) performed in random order for 15 minutes:

1) PEEP set at $5 \mathrm{~cm} \mathrm{H}_{2} \mathrm{O}\left(\mathrm{PEEP}_{\text {low }}\right)$

2) $\mathrm{PEEP}$ set at $15 \mathrm{~cm} \mathrm{H}_{2} \mathrm{O}\left(\mathrm{PEEP}_{\text {high }}\right)$
For safety reasons, in one patient with clinical PEEP of $18 \mathrm{~cm} \mathrm{H}_{2} \mathrm{O}, \mathrm{PEEP}_{\text {low }}$ was set at $10 \mathrm{~cm} \mathrm{H}_{2} \mathrm{O}$ and $\mathrm{PEEP}_{\text {high }}$ at $20 \mathrm{~cm}$ $\mathrm{H}_{2} \mathrm{O}$. FIO $\mathrm{F}_{2}$ during each study phase was titrated to obtain peripheral oxygen saturation greater than or equal to $88 \%$.

\section{Gas Exchange and Ventilation Data}

During the last minutes of each phase, we collected arterial blood gas analysis, and we performed end-inspiratory and endexpiratory occlusions to measure the plateau pressure (Pplat) and the total PEEP $\left(\mathrm{PEEP}_{\text {tot }}\right)$, carefully checking muscles relaxation in patients on pressure support (9). VT as average value from 10 breaths was collected, too. Then, we calculated:

1) The driving pressure (DP) as (Pplat-PEEP $\left.{ }_{\text {tot }}\right)$

2) The respiratory system static compliance (Crs) as VT/DP

3) The ventilatory ratio, as previously described, with higher values indicating less efficient $\mathrm{CO}_{2}$ clearance (10)

\section{Regional Ventilation by EIT}

We horizontally split the EIT images into two contiguous regions of interests of the same size: nondependent from halfway up and dependent from halfway down. Then, from offline analyses of EIT data obtained during the last minutes of each study phase, we measured:

1) The relative pixel-level ventilation $\left(\mathrm{V}_{\text {pixel }}\right)$

2) The percentage of $V_{T}$ distending dependent lung regions at each PEEP level (dorsal fraction of ventilation), with values nearer to $50 \%$ indicating more homogenous ventilation distribution (11)

3) The Global Inhomogeneity (GI) index, with higher values indicating less homogenous ventilation (12)

4) Changes in end-expiratory lung volume at higher PEEP $\left(\Delta \mathrm{EELV}_{\mathrm{EIT}}\right)(13)$

5) Lung recruitment at higher PEEP $\left(\operatorname{RECR}_{\mathrm{EIT}}\right)$ as the increase in EELV measured byEIT minus the change in EELV predicted from the respiratory system compliance measured at $\mathrm{PEEP}_{\text {low }}$ (i.e., $\mathrm{RECR}_{\mathrm{EIT}}=\Delta \mathrm{EELV}_{\mathrm{EIT}}-\triangle \mathrm{EELV}_{\mathrm{Crs}}=$ $\triangle \mathrm{EELV}_{\mathrm{EIT}}-\left[\mathrm{Crs}\right.$ at $\left.\left.\mathrm{PEEP}_{\text {low }} \times \triangle \mathrm{PEEP}\right]\right)(13)$

6) The recruitment to inflation $(\mathrm{R} / \mathrm{I})$ ratio, calculated as the compliance of the recruited lung $\left(\mathrm{C}_{\mathrm{RECR}}=\mathrm{RECR}_{\mathrm{EIT}} / \triangle \mathrm{PEEP}\right)$ divided by the compliance of the baby lung (Crs at PEEP $5 \mathrm{~cm} \mathrm{H} \mathrm{H}_{2} \mathrm{O}$ ). Higher values of the $\mathrm{R} / \mathrm{I}$ ratio indicates larger potential for lung recruitment $(14,15)$

\section{Regional Perfusion by EIT}

In paralyzed patients $(n=7)$, during the PEEP $5 \mathrm{~cm} \mathrm{H}_{2} \mathrm{O}$ step, after assessing lung mechanics, we performed a second endinspiratory occlusion lasting 20 seconds and, 2 seconds after the start, we injected a bolus of $10 \mathrm{~mL}$ of $5 \% \mathrm{NaCl}$ solution via the central venous catheter. Then, from the offline analysis of the EIT data recorded during the saline bolus injection, we measured (Fig. 1):

1) The pixel-level relative regional pulmonary perfusion $\left(Q_{\text {pixel }}\right)$ : the cardiac region was removed from the images and the slope of the EIT signal deflection during the 
saline bolus injection in each pixel was normalized to the overall detected signal, yielding the relative $Q_{\text {pixel }}(16)$

2) Ventilation-perfusion $(V / Q)$ mismatch: if the amount of the $\mathrm{V}_{\text {pixel }}$ or $\mathrm{Q}_{\text {pixel }}$ was less than or equal to $10 \%$ of the highest pixel-level value measured in that patient for that study step, then the pixel was classified as either nonventilated or nonperfused, respectively. Then, V/Q mismatch was quantified as the percentage of pixels that were classified as ventilated but not perfused (dead space fraction) plus the percentage of those perfused but not ventilated (shunt fraction). In this way, V/Q mismatch ranged between $0 \%$ and $100 \%$, with higher values indicating more mismatch

3) To quantify the relative contribution of the dead space versus shunt fraction to V/Q mismatch, we calculated the dead space to shunt ratio as the dead space fraction divided by shunt fraction. Values higher than 1 indicates more relevant role of dead space in determining V/Q mismatch and vice versa

\section{Statistical Analysis}

Sample size was similar to previous physiologic studies $(13,17)$. Comparisons between the two PEEP levels of normally distributed variables were performed by repeated measure $t$ test, while non-normally distributed variables were compared by Wilcoxon signed rank test. Normality was tested by the Shapiro-Wilk test. Association between two variables was assessed by Spearman regression coefficient. A level of $p$ value of less than 0.05 (two-tailed) was considered as statistically significant. Normally distributed data are indicated as mean $\pm \mathrm{SD}$, while median and interquartile range are used to report non-normally distributed variables. Statistical analyses were performed by SigmaPlot 11.0 (Systat Software, San Jose, CA).

\section{RESULTS}

\section{Patients' Characteristics}

Patients were 57 years old (54-64 yr old), four were obese but comorbidities were few. Patients were studied 5 days (1-11 d) after intubation and, under clinical settings, three patients had mild, five patients moderate, and two severe ARDS (Table 1).

\section{Physiologic Effects of PEEP and Potential for Lung Recruitment}

When looking at average values, increasing PEEP by $10 \mathrm{~cm}$ $\mathrm{H}_{2} \mathrm{O}$ did not affect the respiratory mechanics: the respiratory system compliance remained relatively high, keeping the DP below safe threshold despite intermediate VT (Table 2).

At $\mathrm{PEEP}_{\text {high }}$, oxygenation improved, albeit by small extent (Table 2) and arterial $\mathrm{CO}_{2}$ tension significantly increased at constant VT and RR (Table 2) due to significant increase in dead space as assessed by the ventilatory ratio (Table 2). Measures of ventilation inhomogeneity by EIT improved, too: dorsal fraction of ventilation became closer to $50 \%$ and the GI index decreased (Table 2).
At PEEP ${ }_{\text {high }}$, the global increase in lung volume $\left(\triangle \mathrm{EELV}_{\mathrm{EIT}}\right)$ was of $800 \mathrm{~mL}(618-987 \mathrm{~mL})$, of which $309 \mathrm{~mL}(260-487 \mathrm{~mL})$ was due to recruitment $\left(\mathrm{RECR}_{\mathrm{ETT}}\right)$. Potential for lung recruitment measured by EIT through the R/I ratio showed relatively high median values of $0.79(0.53-1.08)$, but with extremely large variability, as indicated by range of 0.16 to 1.40 .

The $\mathrm{R} / \mathrm{I}$ ratio was not correlated with the days of ventilation $(r=0.079 ; p=0.81)$, while we disclosed significant correlation between the R/I ratio and the $\mathrm{FIO}_{2}$ at $\mathrm{PEEP}_{\text {low }}(r=0.603 ; p=0.05)$.

\section{Regional Ventilation-Perfusion Mismatch}

Table 3 summarizes patient-level values of ventilation and perfusion measured by EIT at PEEP $_{\text {low }}$ in seven paralyzed patients. The percentage of pixels with V/Q mismatch was $34 \%(32-45 \%)$ : six out of seven patients had quite large V/Q mismatch with values higher than $30 \%$. The dead space fraction was much more relevant than the shunt fraction in all but one patients and the dead space to shunt ratio was, indeed, 2.3 (1.6-3.9) (Table 3).

\section{DISCUSSION}

Study main findings can be summarized as follows: in a population of 10 patients with ARDS from COVID-19, potential for lung recruitment presents high inter-individual variability; higher R/I ratio values or higher $\mathrm{FIO}_{2}$ 's needed to maintain viable oxygenation at lower PEEP could be used to identify patients with larger potential for lung recruitment; ARDS from COVID-19 is characterized by elevated ventilation-perfusion mismatch, with larger prevalence of ventilated nonperfused lung units (dead space) in comparison to perfused nonventilated units (shunt).

Potential for lung recruitment might be a crucial physiologic measure in ARDS patients (18) for two main reasons. From a clinical perspective, previous studies showed that higher potential for lung recruitment is an independent predictor of mortality in ARDS (18). From a physiologic point of view, application of higher PEEP levels in patients with larger potential for recruitment could enhance lung protection through decreased atelectrauma (19). However, likewise many other pathophysiological features (e.g., hyper- vs hypo-inflammatory condition) (20), previous observations suggested that potential for lung recruitment can be highly variable in ARDS, possibly identifying specific ARDS subphenotypes with higher versus lower recruitability (18). Our population of COVID19 patients with ARDS confirmed that physiologic variability and need for personalized treatment are hallmarks of ARDS (21). Potential for lung recruitment in our population ranged from 0.16 (meaning that the baby lung size increased only by $16 \%$ with a PEEP change of $10 \mathrm{~cm} \mathrm{H}_{2} \mathrm{O}$ ) to 1.40 (with an increase of the baby lung of $140 \%$ ). These data generate the hypothesis that histopathological characteristics of ARDS from COVID-19 may be extremely heterogeneous in terms of the most represented lesions (e.g., interstitial vs intra-alveolar edema, reduced aeration vs fibrosis). This variability may be a consequence of the viral load, of the individual inflammatory response, or a mix of both. Our data suggest that potential 
TABLE 1. Main Characteristics of the Study Population

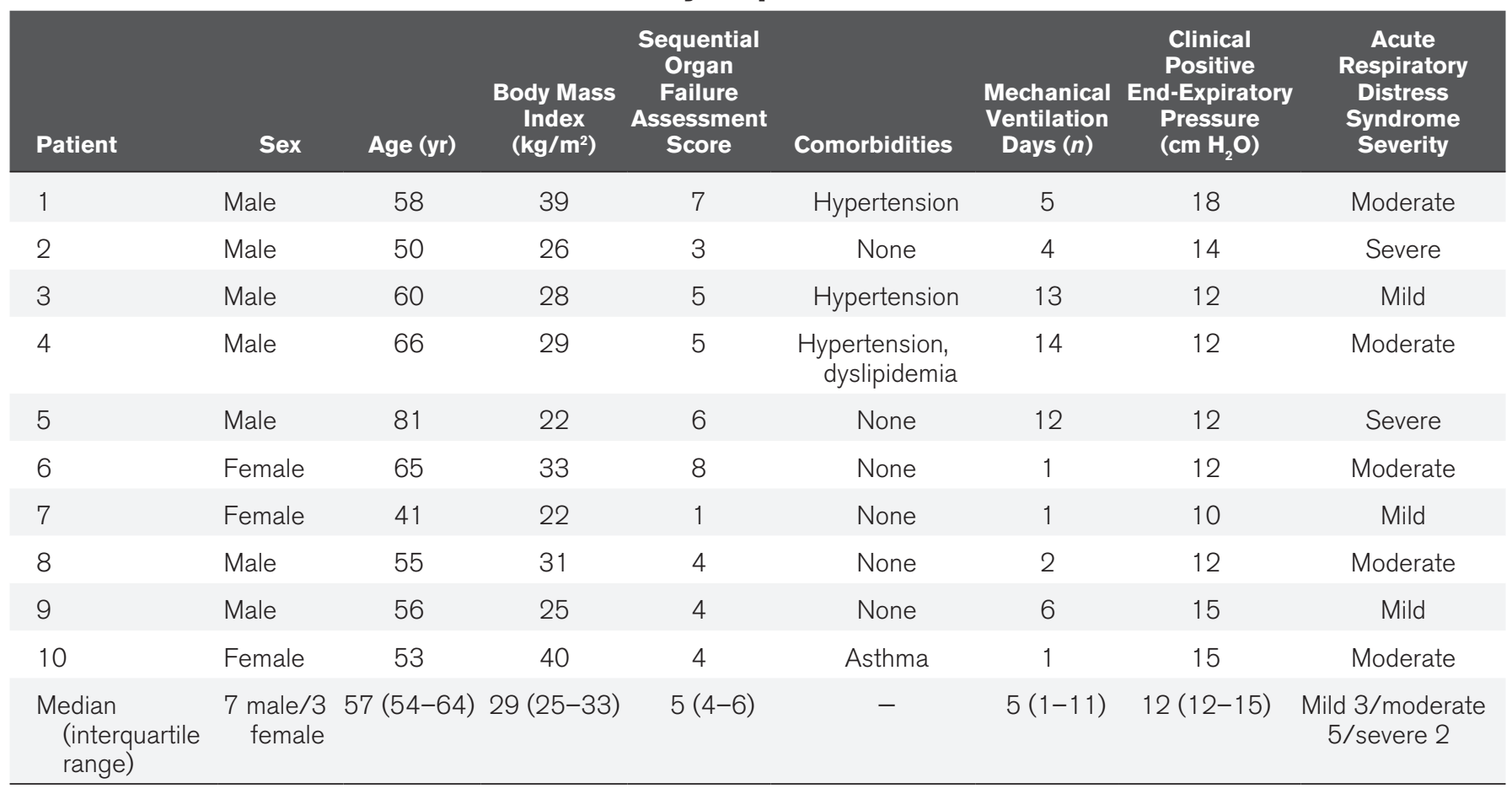

Dash indicates data is not relevant for this variable, which is descriptive and not quantitative.

\section{TABLE 2. Respiratory Physiology and Ventilation Homogeneity Measured by Electrical Impedance Tomography at Higher Versus Lower Positive End-Expiratory Pressure}

\begin{tabular}{|c|c|c|c|}
\hline Variable & $\begin{array}{c}\text { PEEP } P_{\text {low }} \text { at } 5 \mathrm{~cm} \\
\mathrm{H}_{2} \mathrm{O}(n=10)\end{array}$ & $\begin{array}{c}\text { PEE } P_{\text {high }} \text { at } 15 \mathrm{~cm} \\
\mathrm{H}_{2} \mathrm{O}(n=10)\end{array}$ & $p^{a}$ \\
\hline Tidal volume (mL/kg predicted body weight) & $7.4(6.1-7.7)$ & $7.4(6.1-8.6)$ & 0.75 \\
\hline Total PEEP $\left(\mathrm{cm} \mathrm{H}_{2} \mathrm{O}\right)$ & $5(5-6)$ & $15(15-16)$ & $<0.01$ \\
\hline Respiratory rate (breaths/min) & $22(19-25)$ & $23(18-25)$ & 1.00 \\
\hline Plateau pressure $\left(\mathrm{cm} \mathrm{H}_{2} \mathrm{O}\right)$ & $16 \pm 3$ & $28 \pm 3$ & $<0.001$ \\
\hline Driving pressure $\left(\mathrm{cm} \mathrm{H}_{2} \mathrm{O}\right)$ & $10 \pm 2$ & $12 \pm 2$ & 0.07 \\
\hline Respiratory system compliance $\left(\mathrm{mL} / \mathrm{cm} \mathrm{H}_{2} \mathrm{O}\right)$ & $47 \pm 8$ & $43 \pm 11$ & 0.11 \\
\hline $\mathrm{PaO}_{2} / \mathrm{FlO}_{2}$ & $99(69-145)$ & $116(97-196)$ & $<0.01$ \\
\hline $\mathrm{Ph}$ & $7.38 \pm 0.07$ & $7.37 \pm 0.06$ & 0.35 \\
\hline $\mathrm{PaCO}_{2}(\mathrm{~mm} \mathrm{Hg})$ & $50.6 \pm 7.5$ & $55.4 \pm 9.3$ & $<0.01$ \\
\hline Ventilatory ratio & $2.1 \pm 0.5$ & $2.3 \pm 0.6$ & 0.03 \\
\hline Dorsal fraction of ventilation (\%) & $30 \pm 10$ & $39 \pm 15$ & $<0.01$ \\
\hline Global inhomogeneity index (\%) & $70 \pm 11$ & $59 \pm 10$ & 0.001 \\
\hline
\end{tabular}

PEEP $=$ positive end-expiratory pressure.

${ }^{a}$ Normally distributed variables were compared by repeated measure $t$ test, while non-normally distributed variables by Wilcoxon signed rank test.

for lung recruitment should be assessed in each patient with ARDS from COVID-19, for example, to guide personalized titration of PEEP. The R/I ratio can be computed at the bedside by EIT or by other simpler and less expensive methods (14, 15). Alternatively, impairment of oxygenation measured at low PEEP could be used to predict recruitability.
The average physiologic effects of higher PEEP in this population were somehow conflicting: the $\mathrm{R} / \mathrm{I}$ ratio was higher than previously reported in ARDS patients (15), ventilation inhomogeneity decreased and oxygenation improved, but at the same time, the ventilatory ratio increased, suggesting higher dead space. Higher PEEP usually worsens $\mathrm{CO}_{2}$ clearance by causing 
TABLE 3. Ventilation and Perfusion Matching Measured by Electrical Impedance Tomography

\begin{tabular}{lcccc} 
Patient & $\begin{array}{c}\text { Ventilation/Perfusion } \\
\text { Mismatch (\% of Pixels) }\end{array}$ & $\begin{array}{c}\text { Dead Space } \\
\text { Fraction (\% of Pixels) }\end{array}$ & $\begin{array}{c}\text { Shunt Fraction } \\
\text { (\% of Pixels) }\end{array}$ & $\begin{array}{c}\text { Dead Space to } \\
\text { Shunt Ratio }\end{array}$ \\
\hline 2 & 46 & 41 & 5 & 8.2 \\
3 & 43 & 6 & 37 & 0.2 \\
5 & 49 & 35 & 15 & 2.3 \\
6 & 32 & 20 & 13 & 1.5 \\
8 & 34 & 22 & 13 & 1.7 \\
9 & 26 & 18 & 8 & 2.3 \\
Median (interquartile range) & 34 & 27 & 5 & 5.4 \\
\hline
\end{tabular}

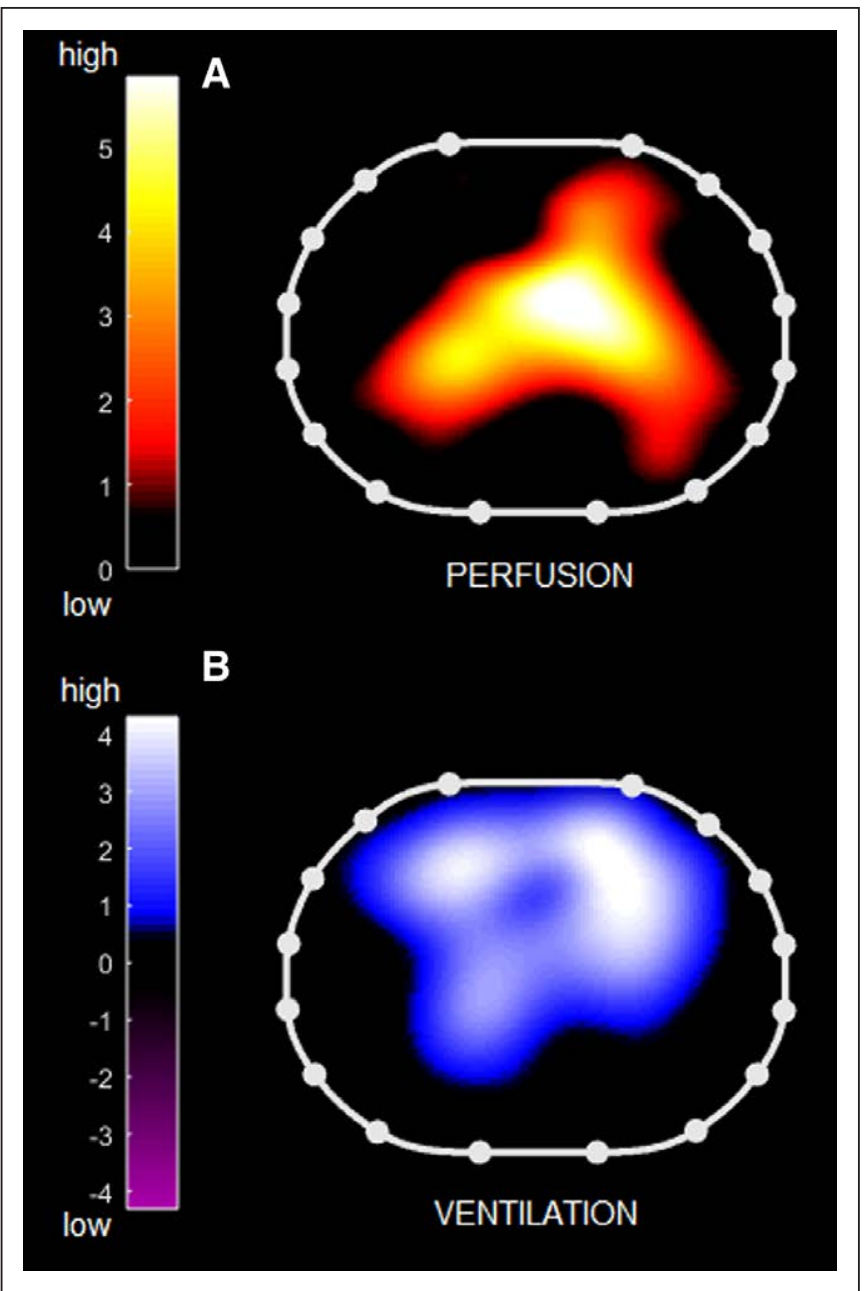

Figure 1. Regional ventilation-perfusion mismatch measured by electrical impedance tomography. Topographic distribution of ventilation (B) and perfusion (A) measured by electrical impedance tomography in a representative patient: note the discrepancy between distribution of ventilated versus perfused lung regions. Right side of the patient is to the left of the images.

regional overdistension and diversion of perfusion from ventilated areas. Of note, overdistension and recruitment coexist to an unpredictable extent in ARDS (22). Presence of vasodilation of pulmonary vessels (7) and micro-thrombosis might have further complicated the dynamics of perfusion redistribution at higher PEEP. Compression of dilated vessels in affected lung regions might represent an alternative to recruitment for explaining the PEEP-induced improvement in oxygenation. Furthermore, higher PEEP could worsen the right heart function, decreasing the cardiac output and reducing shunt (23), further improving oxygenation. Thus, average effects of higher PEEP in this population could already suggest some predominance of detrimental (increased dead space, overdistension, poor right heart function) versus potentially beneficial (recruitment, improved homogeneity) physiologic effects.

Derangements in gas exchange during ARDS are caused by regional mismatch between ventilation and perfusion. In this cohort of COVID-19 patients, ventilation/perfusion mismatch was elevated and mainly due to nonperfused but ventilated units (dead space fraction). These observations add to those from previous studies indicating that elevated $\mathrm{D}$-dimers are an independent predictor of mortality (3) and that pulmonary vessels are enlarged (7) in COVID-19 patients with ARDS. Inflammatory diffuse micro-thrombosis leading to elevated D-dimers, higher pulmonary vascular resistance and larger dead space fraction might be the underlying mechanism of the observed alterations, thus representing a key pathophysiological trait of ARDS from COVID-19. Specific treatments limiting the detrimental effects of dead space ventilation and diffuse micro-thrombosis (e.g., $5 \% \mathrm{CO}_{2}$ inhalation or pulmonary vascular vasodilation) (24) could be tested in these patients.

This study has few limitations: first, the population was small, but we performed multiple refined physiologic measures, and given the rapid spread of COVID-19 cases worldwide, we wanted to present our results early to support formulation of more solid clinical and research hypotheses. Second, the time we left patients at each PEEP level was short, albeit similar to previous studies (13) and longer than the one used in the first study reporting the methodology to assess the R/I ratio (15). Third, patients were studied at different time points along their clinical time course, and this likely corresponded to different 
phases of the inflammation process, further increasing heterogeneity. However, potential for lung recruitment was not correlated with days from intubation, likely indicating that intubation might not be an accurate estimate of the highest severity reached by this syndrome. Fourth, albeit EIT measures of change in lung volumes, recruitment and ventilation-perfusion mismatch have been validated $(13,16)$, only half of the lung parenchyma lies within the imaging field (25). Fifth, we did not perform echocardiography to precisely assess the right heart function and the cardiac output at the two PEEP levels, thus leaving to speculation part of the explanations for our findings.

\section{CONCLUSIONS}

Potential for lung recruitment in patients with ARDS from COVID-19 is highly variable and simple bedside estimates of recruitability should guide personalized mechanical ventilation settings. Elevated ventilation-perfusion mismatch due to high dead space fraction could be a specific characteristic of this syndrome and inform the development of effective treatments.

\section{ACKNOWLEDGMENTS}

We thank Dr. Laurent Brochard for sharing his stimulating ideas and Dr. Luciano Gattinoni for critical revision of the article. We are grateful to Eckhard Teschner from Drägerwerk AG \& Co. KGaA, Lubeck, Germany, for providing the electrical impedance tomography perfusion software tool free of charge.

This study was performed at the Department of Anesthesia, Critical Care and Emergency, Fondazione IRCCS Ca' Granda Ospedale Maggiore Policlinico, Milan, Italy.

Supported, in part, by institutional funding (to Drs. Mauri and Pesenti) from the Department of Anesthesia, Critical Care and Emergency, Fondazione IRCCS Ca' Granda Ospedale Maggiore Policlinico, Milan, Italy.

Dr. Mauri received personal fees from Fisher and Paykel, Draeger Medical, Mindray outside of the present work. Dr. Grasselli received payment for lectures from Draeger Medical, Getinge, Fisher \& Paykel, Biotest, Thermofisher; travel/accommodation/congress registration support from Getinge and Biotest, all outside of the present work. Dr. Pesenti received personal fees from Maquet, Novalung/Xenios, Baxter, and Boehringer Ingelheim, outside the submitted work. The remaining authors have disclosed that they do not have any potential conflicts of interest.

For information regarding this article, E-mail: tommaso.mauri@unimi.it

\section{REFERENCES}

1. Wang D, Hu B, Hu C, et al: Clinical characteristics of 138 hospitalized patients with 2019 novel coronavirus-infected pneumonia in Wuhan, China. JAMA 2020; 323:1061-1069

2. Yang $X, Y u Y, X u J$, et al: Clinical course and outcomes of critically ill patients with SARS-CoV-2 pneumonia in Wuhan, China: A singlecentered, retrospective, observational study. Lancet Respir Med 2020. Feb 24. [online ahead of print]

3. Zhou F, Yu T, Du R, et al: Clinical course and risk factors for mortality of adult in patients with COVID-19 in Wuhan, China: A retrospective cohort study. Lancet 2020; 395:1054-1062

4. Holshue ML, DeBolt C, Lindquist S, et al; Washington State 2019nCoV Case Investigation Team: First case of 2019 novel coronavirus in the United States. N Engl J Med 2020; 382:929-936

5. Spinelli E, Grieco DL, Mauri T: A personalized approach to the acute respiratory distress syndrome: Recent advances and future challenges. J Thorac Dis 2019; 11:5619-5625
6. Shi $\mathrm{H}$, Han $\mathrm{X}$, Jiang $\mathrm{N}$, et al: Radiological findings from 81 patients with COVID-19 pneumonia in Wuhan, China: A descriptive study. Lancet Infect Dis 2020; 20:425-434

7. Albarello F, Pianura E, Di Stefano F, et al; COVID 19 INMI Study Group: 2019-novel Coronavirus severe adult respiratory distress syndrome in two cases in Italy: An uncommon radiological presentation. Int J Infect Dis 2020; 93:192-197

8. Li Y, Xia L: Coronavirus disease 2019 (COVID-19): Role of chest CT in diagnosis and management. AJR Am J Roentgenol 2020; 4:1-7

9. Bellani G, Grassi A, Sosio S, et al: Plateau and driving pressure in the presence of spontaneous breathing. Intensive Care Med 2019; 45:97-98

10. Sinha P, Calfee CS, Beitler JR, et al: Physiologic analysis and clinical performance of the ventilatory ratio in acute respiratory distress syndrome. Am J Respir Crit Care Med 2019; 199:333-341

11. Yoshida T, Piraino T, Lima CAS, et al: Regional ventilation displayed by electrical impedance tomography as an incentive to decrease positive end-expiratory pressure. Am J Respir Crit Care Med 2019; 200:933-937

12. Zhao Z, Möller K, Steinmann D, et al: Evaluation of an electrical impedance tomography-based Global Inhomogeneity Index for pulmonary ventilation distribution. Intensive Care Med 2009; 35:1900-1906

13. Mauri T, Eronia N, Turrini C, et al: Bedside assessment of the effects of positive end-expiratory pressure on lung inflation and recruitment by the helium dilution technique and electrical impedance tomography. Intensive Care Med 2016; 42:1576-1587

14. Chen L, Chen GQ, Shore K, et al: Implementing a bedside assessment of respiratory mechanics in patients with acute respiratory distress syndrome. Crit Care 2017; 21:84

15. Chen L, Del Sorbo L, Grieco DL, et al: Potential for lung recruitment estimated by the recruitment-to-inflation ratio in acute respiratory distress syndrome. A clinical trial. Am J Respir Crit Care Med 2020; 201:178-187

16. Bluth $T$, Kiss $T$, Kircher M, et al: Measurement of relative lung perfusion with electrical impedance and positron emission tomography: An experimental comparative study in pigs. Br J Anaesth 2019; 123:246-254

17. Mauri T, Bellani G, Confalonieri A, et al: Topographic distribution of tidal ventilation in acute respiratory distress syndrome: Effects of positive end-expiratory pressure and pressure support. Crit Care Med 2013; 41:1664-1673

18. Gattinoni L, Caironi $\mathrm{P}$, Cressoni M, et al: Lung recruitment in patients with the acute respiratory distress syndrome. N Engl J Med 2006; 354:1775-1786

19. Caironi $P$, Cressoni M, Chiumello D, et al: Lung opening and closing during ventilation of acute respiratory distress syndrome. Am J Respir Crit Care Med 2010; 181:578-586

20. Sinha P, Delucchi KL, McAuley DF, et al: Development and validation of parsimonious algorithms to classify acute respiratory distress syndrome phenotypes: A secondary analysis of randomised controlled trials. Lancet Respir Med 2020; 8:247-257

21. Constantin JM, Jabaudon M, Lefrant JY, et al; AZUREA Network: Personalised mechanical ventilation tailored to lung morphology versus low positive end-expiratory pressure for patients with acute respiratory distress syndrome in France (the LIVE study): A multicentre, single-blind, randomised controlled trial. Lancet Respir Med 2019; 7:870-880

22. Puybasset L, Gusman P, Muller JC, et al: Regional distribution of gas and tissue in acute respiratory distress syndrome. III. Consequences for the effects of positive end-expiratory pressure. CT Scan ARDS Study Group. Adult Respiratory Distress Syndrome. Intensive Care Med 2000; 26:1215-1227

23. Lynch JP, Mhyre JG, Dantzker DR: Influence of cardiac output on intrapulmonary shunt. J Appl Physio/ 1979; 46:315-321

24. Kolobow T, Spragg RG, Pierce JE: Massive pulmonary infarction during total cardiopulmonary bypass in unanesthetized spontaneously breathing lambs. Int J Artif Organs 1981; 4:76-81

25. Spinelli E, Mauri T, Fogagnolo A, et al: Electrical impedance tomography in perioperative medicine: Careful respiratory monitoring for tailored interventions. BMC Anesthesiol 2019; 19:140 